\title{
A model for vortex ring formation in a starting buoyant plume
}

\author{
By MICHAEL SHUSSER AND MORTEZA GHARIB \\ Graduate Aeronautical Laboratories, California Institute of Technology, Pasadena, \\ MC 205-45, CA 91125, USA
}

(Received 20 February 1999 and in revised form 3 March 2000)

Vortex ring formation in a starting axisymmetric buoyant plume is considered. A model describing the process is proposed and a physical explanation based on the Kelvin-Benjamin variational principle for steady vortex rings is provided. It is shown that Lundgren et al.'s (1992) time scale, the ratio of the velocity of a buoyant plume after it has travelled one diameter to its diameter, is equivalent to the time scale (formation time) proposed by Gharib et al. (1998) for uniform-density vortex rings generated with a piston/cylinder arrangement. It is also shown that, similarly to piston-generated vortex rings (Gharib et al. 1998), the buoyant vortex ring pinches off from the plume when the latter can no longer provide the energy required for steady vortex ring existence. The dimensionless time of the pinch-off (the formation number) can be reasonably well predicted by assuming that at pinch-off the vortex ring propagation velocity exceeds the plume velocity. The predictions of the model are compared with available experimental results.

\section{Introduction}

A vortex ring can be generated in the laboratory by the motion of a piston pushing a column of a fluid of length $L$ through an orifice or nozzle of diameter $D$. This process results in the separation of the boundary layer at the edge of the orifice or nozzle. Subsequently the separated boundary layer rolls up into a spiral.

The piston/cylinder arrangement has been extensively used to address the problem of vortex ring formation (Shariff \& Leonard 1992; Lim \& Nickels 1995). Recently Gharib, Rambod \& Shariff (1998) in their experimental study of vortex ring formation addressed the question of the largest circulation that a vortex ring can attain, by increasing $L / D$ while keeping the average piston velocity fixed.

Gharib et al. (1998) showed that two distinct states of the flow exist for a wide range of the ratio of piston stroke to diameter $(L / D)$ or 'formation times'. Whereas for small stroke ratios only a single vortex ring was observed, the flow field generated by large $L / D$ values always resulted in a leading vortex ring followed by a trailing jet. Comparing the total circulation produced by the motion of the piston with that of the resulting vortex ring, they were able to define the time of the transition between these two flow states, i.e. when the vortex ring pinches off from its generating axisymmetric jet.

It turned out that pinch-off was always observed to occur at a stroke ratio (formation time) of approximately 4 . This universal time scale was called the 'formation number'. The universality of this number was tested by generating vortex rings with different jet exit diameters, Reynolds numbers and exit boundary conditions, as well as with various non-impulsive piston velocities programs. 
The existence of the formation number was shown to be consistent with the KelvinBenjamin variational principle for steady axis-touching vortex rings (Kelvin 1880, $\S 18$; Benjamin 1976). According to this principle, a steady translating vortex ring has maximum energy with respect to impulse-preserving iso-vortical (i.e. preserving the circulation of each fluid element) perturbations. It has been used in the mathematical literature for investigating the stability and existence of vortex-ring-type solutions (Friedman \& Turkington 1981; Amick \& Fraenkel 1986; Wan 1988). It follows from this principle that the pinch-off occurs when the apparatus is no longer able to deliver the energy required for the existence of a steady vortex ring. Gharib et al. (1998) demonstrated that, based on the measured impulse, circulation and energy of the observed vortex rings, the Kelvin-Benjamin variation principle correctly predicts the range of observed formation numbers. Later Mohseni \& Gharib (1998) and Shusser \& Gharib (2000) used the Kelvin-Benjamin principle to model the vortex ring formation process.

In uniform-density fluids, the piston motion provides the energy required for the vortex ring formation. Another way to produce a vortex ring is by releasing a lighter or heavier fluid into a different ambient fluid. In this case, the energy is provided by the action of the buoyancy force. Such a ring is, therefore, called a buoyant vortex ring (Turner 1957, 1973). Depending on whether the buoyancy is supplied steadily from a maintained source or remains confined to a limited volume of the fluid, the physical situation can be described as a starting buoyant plume or a buoyant thermal respectively (Turner 1973, pp. 165-166).

Turner (1957) pointed out that if a vortex ring contains fluids other than the surrounding fluid, it may show a distinct variation in its behaviour. Therefore, it is natural to suspect such variations to exist in the early formation stages of buoyant vortex rings. Also, we are interested to see whether this variation would alter the formation number, as defined by Gharib et al. (1998), and applicability of the Kelvin-Benjamin principle to buoyant vortex rings. These questions provided the basis for the present work.

Buoyant plumes (Morton, Taylor \& Turner 1956; Turner 1962, 1973; Moses, Zocchi \& Libchaber 1993) and buoyant vortex rings (Turner 1957, 1973; Lundgren \& Mansour 1991) have been the subject of numerous studies. Older works were reviewed by Fay (1973), List (1982) and Gebhart, Hilder \& Kelleher (1984). Shlien \& Boxman (1981) measured the temperature field in a laminar starting plume. Tanny \& Shlien (1985) and Chay \& Shlien (1986) used digital processing to measure the velocity and scalar (heat or species concentration) fields of a laminar starting plume. Moses et al. (1993) presented an experimental study of the scaling laws for a laminar starting plume. Berezin \& Hutter (1995) modelled an axisymmetric plume subjected to an external vortex. Friedl, Härtel \& Fanneløp (1999) conducted an experimental study of starting plumes over area sources. Starting plumes in rotating fluids were investigated by Fernando, Chen \& Ayotte (1998) and by Bush \& Woods (1999), while compressibility effects are studied in Rast (1998).

However, these studies did not devote much attention to the problem of vortex ring formation and pinch-off in a starting buoyant plume. Moses et al. (1993) did not observe vortex ring formation due to high viscosity of their fluids. Shlien \& Boxman (1981) and Chay \& Shlien (1986) did not measure vorticity fields, while Tanny \& Shlien (1985) measured the vorticity only in the cap of the plume. Fernando et al. (1998) did not report any quantitative data for the first $3 \mathrm{~s}$ of plume development, i.e. until well after the completion of pinch-off, as we shall see later. In Friedl et al. (1999)'s experiments the buoyant fluid was injected into the heavier medium with a finite velocity. 
Based on the above studies, one can infer the existence of the pinch-off phenomenon only from indirect signs, such as changes in the plume behaviour. For example, Fernando et al. (1998, p. 2371) mention that the plume front first accelerates for a short period and then decelerates continuously. The only exception is a recent numerical work on the dynamics of a plane compressible plume in a stratified layer by Rast (1998). The pinch-off of the generated ring is seen very clearly in his figures 3 and 8 . On the other hand, vortex ring formation in a starting axisymmetric buoyant plume and its pinch-off from the generating plume had not been addressed until the recent experimental study of Johari \& Gharib (1998).

Turner (1962) showed that a starting buoyant plume consists of a 'cap', which is its advancing front, and a 'stem', which is the rest of the plume excluding the region in the immediate vicinity of the heat source. The vortex ring is formed in the cap. The source of the vorticity to the ring is the thin mixing layer formed at the interface between the different-density fluids around the stem. Similarity solutions describing the flow field in both parts of the plume were obtained by Turner (1962). These steady-state solutions, however, are valid only after vortex ring formation is completed.

Especially relevant to the present study is the work of Lundgren, Yao \& Mansour (1992) who studied a downburst by conducting experimental and numerical investigation of the vortex ring formation in buoyant thermals. By assuming the Boussinesq approximation, they showed that the solution to this problem is determined by two characteristic parameters: the radius of the thermal $R_{0}$ and a characteristic time

$$
T_{0}=\sqrt{\frac{R_{0} \rho}{g \Delta \rho}},
$$

where $g$ is the gravitational acceleration, $\Delta \rho=\rho_{0}-\rho$, and $\rho, \rho_{0}$ are the densities of the plume fluid and ambient fluid, respectively. We will consider the relationship between Lundgren et al.'s (1992) characteristic time $T_{0}$ and the formation number in $\S 4$.

Lundgren et al. (1992) found that the circulation of a moving thermal develops rapidly to a constant value through the formation of a vortex ring. Some important observations can be made from the flow field of the developing vortex ring calculated by Lundgren et al. (1992, figure 7): the generation of vorticity and circulation precede the onset of the ring formation, and at the time of approximately $3 T_{0}$ (their figure $7 a$ ) a region of strong circulating flow appears in the cap area. However, characteristic features of a vortex ring, such as a distinct core, are not seen until later times (their figure $7 c$ ).

Following Gharib et al. (1998), we shall use Lundgren et al.'s (1992) experimental and numerical results to model buoyant vortex ring formation. To begin with, we put forward a hypothesis that the process of buoyant vortex ring formation consists of two stages: the continuous creation of the circulation and formation of the initial vortex ring (stage I) followed by the growth of the ring through accumulation of the generated circulation (stage II). We shall use Lundgren et al.'s (1992) data for the modelling of the first stage (see $\S 5$ ). In $\S 6$, based on their data we estimate buoyant vortex ring energy. We also adopt the assumptions made in Lundgren et al. (1992), as mentioned at the end of $\S 3$.

At time of the submission of this paper, no experimental data were available to validate the proposed model, except the study of Johari \& Gharib (1998). We therefore presented our model as a basis for future experimental work. However, since then an experimental investigation by Pottebaum, Shusser \& Gharib (1999) has further confirmed the validity of the present model. 
(a)

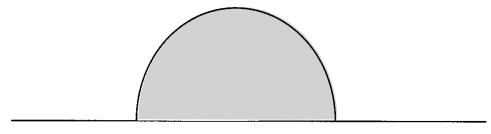

(c)

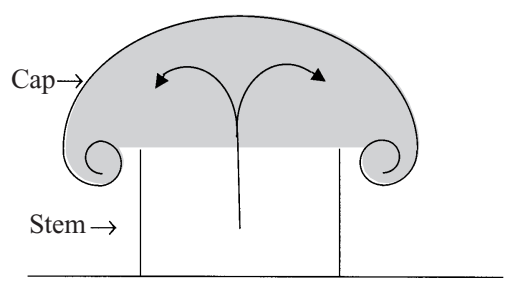

(b)

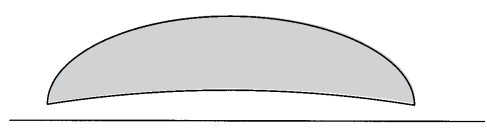

(d)
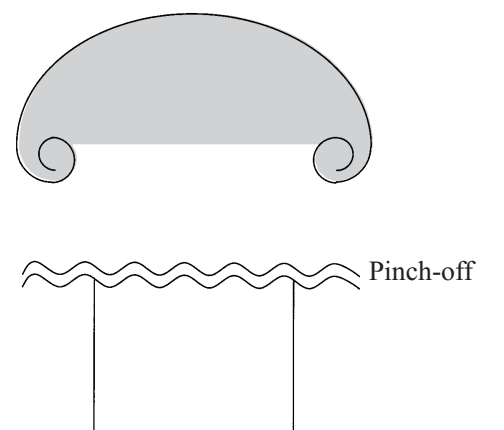

FIGURE 1. The different stages of vortex ring formation: $(a)$ initial mass of a light fluid; $(b)$ formation of the initial ring (stage I); $(c)$ growth of the ring by flow from the stem (stage II); $(d)$ pinch-off of the vortex ring.

\section{Objectives}

The main purpose of the present study is to investigate the formation dynamics of vortex rings in starting buoyant plumes. We are especially interested in verifying the applicability of the Kelvin-Benjamin principle to buoyancy-driven flows.

We propose the following approach to tackling this problem. Following the approach taken by Turner (1962), we spatially divide the plume into the cap and the stem regions. Then, we adopt the aforementioned two-stage hypothesis by considering the initial formation of the ring-like structure in the plume cap as stage I and the growth of this early vortex ring by accumulation of vorticity from the stem as stage II (see figure 1).

During the first stage the form of the cap changes (Turner 1962; Johari \& Gharib 1998). We use the buoyancy force that acts on the lighter fluid to determine the impulse, the kinetic energy and the velocity of the cap. This information will provide the initial conditions for the second stage of the process.

The duration of the first stage, in which most of the circulation is generated and the initial vortex ring starts to form, cannot be calculated analytically. However, it is possible to estimate this time from the results of Lundgren et al. (1992). By analysing the temporal behaviour of the circulation of the thermal (Lundgren et al. 1992, figure 14) and of its flow field (Lundgren et al. 1992, figure 7) one can conclude that this time is approximately $3 T_{0}$. We shall use this information for calculating the initial conditions for stage II in $\S 5$ (see equation (28)).

In the second stage of the process the vortex ring properties are determined by the flux from the stem and the action of the buoyancy force on the cap. The latter can be calculated directly, while we obtain the former by considering the flow in the stem as a buoyant plume from a maintained source (Morton et al. 1956). Thus, all the properties of the vortex ring can be calculated. 
Utilizing the Kelvin-Benjamin principle we then assume that the ring pinches off from the stem when the latter can no longer provide the energy required for steady vortex ring existence. This energy can be computed using the data of Lundgren et al. (1992). Comparing both energies we shall verify the consistency of this approach and estimate the time of pinch-off.

The plan of the paper is as follows. The physical situation is depicted in $\S 3$. The equivalence of the characteristic times for buoyant and non-buoyant vortex rings is shown in $\S 4$. In $\S 5$ we analyse a starting buoyant plume and model its properties. The energy of a buoyant vortex ring is calculated in $\S 6$ using the experimental and numerical data of Lundgren et al. (1992). These results are used in $\S 7$ to estimate the dimensionless energy of the vortex ring at pinch-off and the formation number.

\section{Physical situation}

Let an initial axisymmetric mass of a light fluid be created through a thermodynamic event, e.g. by a heat source. We consider a light (or heavy for a downburst) fluid of density $\rho$ occupying a hemisphere of a radius $R_{0}$ and mass $M=2 M_{0} / 3$, where $M_{0}=\pi \rho R_{0}^{3}$, within a heavier ambient fluid of density $\rho_{0}\left(\rho<\rho_{0}\right)$ (see figure $\left.1 a\right)$. We take the origin of the vertical coordinate $z$ at the source so at the initial moment of time $t=0$ the centre of the hemisphere was at $z=0$ and its impulse $I$, kinetic energy $E$, circulation $\Gamma$ and velocity $U$ were all equal to zero.

Buoyancy force causes the lighter fluid to rise. This motion causes deformation of the hemisphere, creation of circulation and formation of the initial vortex ring (stage I-see figure $1 b$ ). Continuing action of the heat source then creates a rising flow of the lighter fluid (the stem of the plume) following the ring (the cap of the plume) - see figure $1(c)$. The mass flux from the stem into the ring continues until the latter has grown large enough to pinch off, as shown in figure $1(d)$.

Following Lundgren et al. (1992), we assume that both fluids are incompressible and inviscid except for the thin shear layer at the interface and neglect at both stages entrainment of the outer fluid to the stem. We also assume a constant velocity across the stem (a 'top-hat' profile). The latter assumption has been widely used in theoretical studies of buoyant plumes and thermals (Turner 1973).

\section{Characteristic time for buoyant vortex ring formation}

To compare formation times for buoyant and non-buoyant vortex rings, one must ensure that their definitions are consistent in both cases. Hence, we have to compare the characteristic time scale for buoyant vortex ring formation $T_{0}$ and the characteristic time for vortex ring formation by the piston/cylinder arrangement defined by Gharib et al. (1998) as

$$
T_{1}=D / U_{p},
$$

where $D$ is the diameter of the cylinder and $U_{p}$ is the piston velocity.

Equation (2) defines the characteristic time as the ratio of the characteristic length $(D)$ to the characteristic velocity $\left(U_{p}\right)$. We now show that the same definition in the buoyant case results in Lundgren et al.'s time scale (1).

The characteristic length for the buoyant plume problem is the diameter of the thermal $2 R_{0}$, which is equivalent to the diameter of the cylinder for a non-buoyant ring. In this we have assumed that the diameter of the stem is approximately equal to the diameter of the thermal. We shall expand on this assumption in $\S 5.2$. The 
characteristic velocity in the buoyant case is not independent of the characteristic length, which is due to the velocity of the thermal being a function of its position. In this respect, the characteristic velocity must be defined as the velocity $V$ acquired by the thermal after it has travelled one characteristic length, which is

$$
V=\sqrt{4 g R_{0} \Delta \rho / \rho} .
$$

Now, defining the characteristic time scale as the ratio of the characteristic length to the characteristic velocity, i.e.

$$
T_{0}=2 R_{0} / V,
$$

and substituting (3) in (4), we arrive in Lundgren et al.'s time scale for buoyant vortex rings as given by equation (1).

This result shows that the processes of formation of buoyant and non-buoyant vortex rings are closely related. Hence, one can extend the assumptions made by Gharib et al. (1998), Mohseni \& Gharib (1998) and Shusser \& Gharib (2000) for modelling the buoyant vortex ring formation. These assumptions include the slug-flow model (Shariff \& Leonard 1992; Lim \& Nickels 1995) or approximating the ring as a member of Norbury (1973)'s family of vortex rings (Mohseni \& Gharib 1998).

\section{Flow in the plume}

\subsection{Stage I}

The first stage of the process (the generation of circulation) is motion of a constant mass of a light fluid caused by the buoyancy force. The motion is described by the following equations:

$$
\begin{gathered}
\frac{\mathrm{d} M}{\mathrm{~d} t}=0, \\
\frac{\mathrm{d} I}{\mathrm{~d} t}=\frac{\Delta \rho}{\rho} M g, \\
\frac{\mathrm{d} E}{\mathrm{~d} t}=\frac{\Delta \rho}{\rho} M g U, \\
\frac{\mathrm{d} z}{\mathrm{~d} t}=U, \\
U=\frac{I}{M} .
\end{gathered}
$$

Equation (5) follows from the no-entrainment assumption, (6) and (7) give the impulse and energy change under the action of the buoyancy force, while (8) and (9) are auxiliary relationships among the cap velocity, impulse and position. Using (5)-(9) one can obtain all the necessary information about this stage.

\subsection{Stage $I I$}

For the second stage, following Morton et al. (1956, p. 5), one can write for the mass and momentum conservation, respectively

$$
\begin{gathered}
\frac{\mathrm{d}}{\mathrm{d} z}\left(\pi b^{2} U \rho\right)=0, \\
\frac{\mathrm{d}}{\mathrm{d} z}\left(\pi b^{2} U^{2} \rho\right)=\pi b^{2} g \Delta \rho,
\end{gathered}
$$




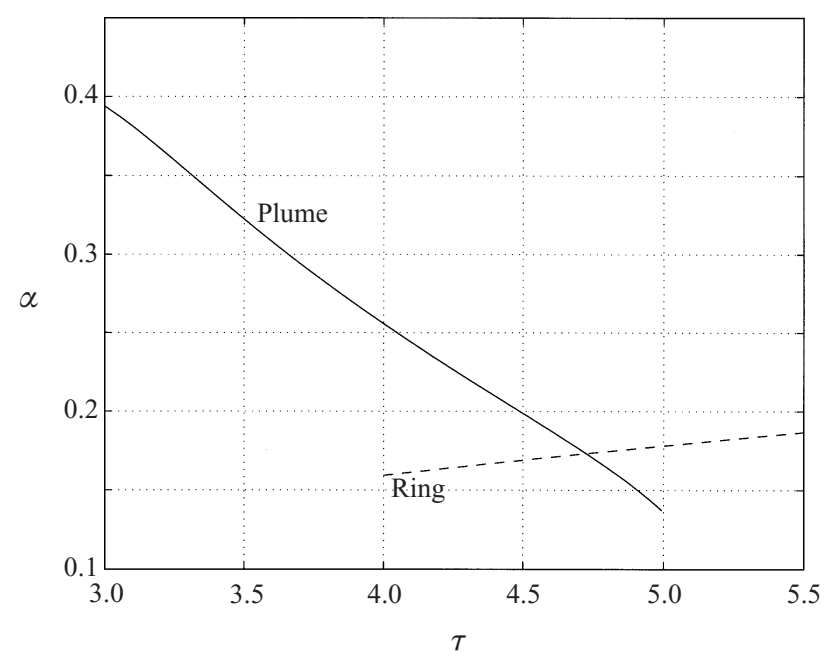

FIGURE 2. Dimensionless energy of the ring and the plume.

where $b$ is the radius of the stem. Equation (10) shows that without entrainment mass flux remains constant along the stem and (11) is the increase in momentum caused by the buoyancy force.

Eliminating $b$ from (10) and (11) and solving the resulting differential equation for $U$ results in

$$
U=\sqrt{\frac{2 g \Delta \rho}{\rho} z .}
$$

It follows from (10) and (12) that the stem radius $b$ changes slowly as $z^{-1 / 4}$ except for the singularity at $z=0$ which corresponds to the influx of cold ambient fluid. In real flow the decrease in the stem radius is cancelled out by the action of entrainment. Therefore, it is possible to neglect the variation of the stem radius during the early stages of plume development, as indeed was obtained experimentally (see figure 2 of Johari \& Gharib 1998). Assuming that the stem radius near the ring remains close to $R_{0}$ and calculating the properties of the ring as in (5)-(9) but adding the flux from the stem, results in

$$
\begin{gathered}
\frac{\mathrm{d} M}{\mathrm{~d} t}=\pi R_{0}^{2} \rho(U-W), \\
\frac{\mathrm{d} I}{\mathrm{~d} t}=\pi R_{0}^{2} \rho U(U-W)+\frac{\Delta \rho}{\rho} M g, \\
\frac{\mathrm{d} E}{\mathrm{~d} t}=\frac{1}{2} \pi R_{0}^{2} \rho U^{2}(U-W)+\frac{\Delta \rho}{\rho} M g W, \\
\frac{\mathrm{d} z}{\mathrm{~d} t}=W .
\end{gathered}
$$

Here $W$ is the propagation velocity of the ring.

To calculate the circulation of the vortex ring we use the slug-flow model (Shariff \& Leonard 1992; Lim \& Nickels 1995),

$$
\frac{\mathrm{d} \Gamma}{\mathrm{d} t}=\frac{1}{2} U^{2} .
$$


Equation (17) has been shown to be a good estimate even for flows with rapidly curving streamlines provided the breadth of the vortex sheet is small compared with its radius of curvature (Sarpkaya 1975, p. 115; Fage \& Johansen 1928). This justifies using (17) for both stages of the process. It should be noted that it is obtained by integrating the vorticity in the mixing layer between the light and ambient fluid. Therefore, for buoyant flows it indirectly accounts for baroclinic vorticity production.

To estimate the propagation velocity we approximate the ring as a member of Norbury's (1973) family of vortex rings. Although Norbury's solutions are for nonbuoyant vortex rings one can expect this to be a reasonable approximation, especially when density differences are small. Using second-order formulae for these rings (Fraenkel 1972, p. 132) one can write

$$
W=\frac{1}{4} \sqrt{\frac{\rho \Gamma^{3}}{\pi I}\left(1+\frac{3}{4} \varepsilon^{2}\right)}\left[\ln \frac{8}{\varepsilon}-\frac{1}{4}+\frac{3 \varepsilon^{2}}{8}\left(\frac{5}{4}-\ln \frac{8}{\varepsilon}\right)\right] .
$$

Here $\varepsilon$ is the cross-section parameter (dimensionless mean core radius) defined in Fraenkel (1972).

Norbury's family has been used for vortex ring modelling by Mohseni \& Gharib (1998). They showed that an appropriate value of $\varepsilon$ is 0.3 . Then

$$
W=0.7658 \sqrt{\frac{\rho \Gamma^{3}}{\pi I}} .
$$

Defining dimensionless variables

$$
\begin{gathered}
\tau=\frac{t}{T_{0}}, \quad \xi=\frac{z}{R_{0}}, \quad m=\frac{M}{M_{0}}, \quad i=\frac{I T_{0}}{M_{0} R_{0}}, \quad e=\frac{E T_{0}^{2}}{M_{0} R_{0}^{2}}, \quad \gamma=\frac{\Gamma T_{0}}{R_{0}^{2}}, \\
u=\frac{U T_{0}}{R_{0}}, \quad w=\frac{W T_{0}}{R_{0}},
\end{gathered}
$$

and choosing $\xi$ as an independent variable the following system of equations is obtained:

$$
\begin{gathered}
\frac{\mathrm{d} \tau}{\mathrm{d} \xi}=\frac{1}{w}, \\
\frac{\mathrm{d} m}{\mathrm{~d} \xi}=\frac{u}{w}-1, \\
\frac{\mathrm{d} i}{\mathrm{~d} \xi}=u\left(\frac{u}{w}-1\right)+\frac{m}{w}, \\
\frac{\mathrm{d} e}{\mathrm{~d} \xi}=\frac{1}{2} u^{2}\left(\frac{u}{w}-1\right)+m, \\
\frac{\mathrm{d} \gamma}{\mathrm{d} \xi}=\frac{1}{2} \frac{u^{2}}{w} \\
u=\sqrt{2 \xi}, \\
w=\frac{0.7658}{\pi} \sqrt{\frac{\gamma^{3}}{i}} .
\end{gathered}
$$


The initial conditions for (21)-(27) are obtained by integrating the first-stage equations (5)-(9) and taking their solution at $3 T_{0}$ :

$$
\tau=3, \quad m=\frac{2}{3}, \quad i=2, \quad e=3, \quad \gamma=\frac{9}{2} \quad \text { when } \quad \xi=\frac{9}{2} .
$$

\section{Buoyant vortex ring energy}

To apply the Kelvin-Benjamin principle one needs to know the energy of a buoyant vortex ring. Following Gharib et al. (1998) we characterize the properties of the ring by considering the dimensionless energy

$$
\alpha=\frac{E}{\sqrt{\rho I \Gamma^{3}}} .
$$

In this section we shall estimate the value of $\alpha$ for a buoyant vortex ring using the data of Lundgren et al. (1992). They consider vortex ring formation in a buoyant thermal. In this problem the flow from the stem does not exist and, hence, we can consider both stages together.

Under the assumption of no entrainment and incompressibility the volume of the thermal remains constant during the process. Therefore, the buoyancy force will remain constant and equal to

$$
F=\frac{4}{3} \pi R_{0}^{3} \Delta \rho g .
$$

Lundgren et al. (1992, p. 465) showed that the viscous drag does not exceed $1 \%$ of the buoyancy force and is, therefore, negligible. Then, the impulse of the ring is

$$
I=F T .
$$

On the other hand, the form of the thermal and, therefore, its added mass change during the motion. However, knowing the impulse of the ring and its velocity we can calculate the energy as

$$
E=\frac{1}{2} I U
$$

According to Lundgren et al. (1992) the circulation of the ring formed is

$$
\Gamma=5 R_{0}^{2} / T_{0} \text {. }
$$

The time at which the circulation of the ring has reached this value can be considered to be the formation time of a vortex ring $(T)$. It depends on the value of characteristic parameter $T_{0}$. We therefore write

$$
T=N T_{0},
$$

without specifying the value of $N$ at this stage.

Substituting in (29) one obtains

$$
\alpha=\frac{u}{5} \sqrt{\frac{\pi N}{15}} .
$$

Calculating the dimensionless velocity $u$ from curves $(a)$ and $(b)$ of figure 14 in Lundgren et al. (1992) and taking the average value we arrive at $u \approx 0.87$. Then

$$
\alpha \approx 0.07963 \sqrt{N} \text {. }
$$




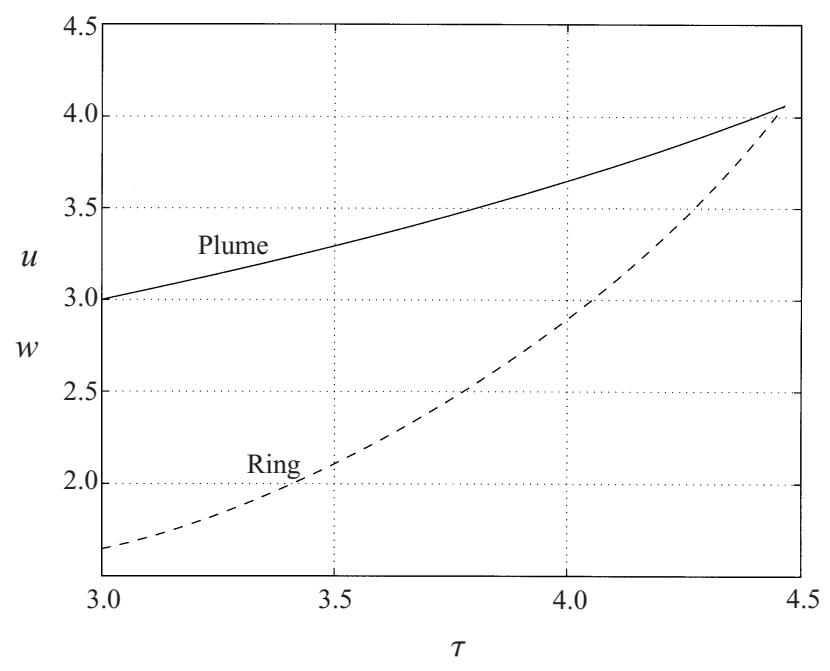

FIGURE 3. Buoyant vortex ring and plume velocities.

\section{Results and discussion}

The time dependence of $\alpha$ was calculated from (36) for the ring and by solving (21)-(28) for the plume. The results are shown in figure 2. At the outset the energy provided by the plume is seen to be larger than the energy required for the ring. As time proceeds the difference becomes smaller until at a certain critical value $(\tau \approx 4.73)$ both energies are equal. The formation of a buoyant vortex ring with more energy is, therefore, impossible. The same phenomenon was obtained by Gharib et al. (1998) for piston-generated vortex rings.

One can conclude that the Kelvin-Benjamin variational principle remains valid for buoyant vortex rings. The critical value of 4.73 corresponds to the formation number for a buoyant vortex ring. It is larger than the value of 4 obtained in Gharib et al. (1998). This can be explained by the fact that for a buoyant vortex ring there are two physical mechanisms providing the energy to the ring: the flux from the plume stem and the action of the buoyancy force. The latter mechanism does not exist for non-buoyant rings and, therefore, according to the Kelvin-Benjamin principle their formation terminates earlier. If this conjecture is correct then one can expect buoyant vortex rings to be thicker than non-buoyant rings created under the same conditions. This is worth experimental study.

The impulse and the circulation for the buoyant ring are also larger than for the non-buoyant one. Therefore, though the energy of the former is larger the value of the dimensionless energy $\alpha$ for this ring is smaller: from figure 2 one sees that $\alpha \approx 0.17$ while Gharib et al. (1998) obtained $\alpha \approx 0.33$.

It is worth noting that Lundgren et al. $(1992$, p. 282) state that the circulation of the ring reaches its final value at about $3 T_{0}$. On the other hand, one can see from their figure 14 that this occurs between $4 T_{0}$ and $5 T_{0}$, in good agreement with our results.

Shusser \& Gharib (2000) demonstrated that another manifestation of the KelvinBenjamin principle can be presented by assuming that pinch-off occurs when the propagation velocity of the ring becomes equal to the flow velocity in the plume, so that mass flux from the stem is no longer possible. To verify this hypothesis we plot in figure 3 the velocities of the plume stem and of the ring calculated by solving (21)-(28). The vortex ring velocity is seen to be initially smaller than the velocity 
in the stem but grows faster until both velocities become equal. This happens when $\tau \approx 4.46$ and can be considered as an approximation for the pinch-off time.

It is important to note that the pinch-off time predicted from comparison of plume and ring velocities $(\tau \approx 4.46)$ is close to the value obtained by considering the energies $(\tau \approx 4.73)$, the difference being $6 \%$. One can conclude that the hypothesis of the equality of ring and plume velocities at pinch-off is equivalent to the KelvinBenjamin principle in the buoyant case, too.

So far, we have taken the duration of the first stage to be $3 T_{0}$, as was obtained by Lundgren et al. (1992). To verify the influence of this parameter we also tried other values by varying the conditions (28). For a duration of $2 T_{0}$ the qualitative picture remains the same, though the numerical values change. Similarly, in this case, energy provided by the plume is initially larger than the energy needed for the ring, and the flow velocity in the plume stem is larger than the vortex ring propagation velocity. The predicted times for pinch-off in this case are $\tau \approx 6.33$ from the Kelvin-Benjamin principle and $\tau \approx 6.77$ from the comparison of the velocities. These values are close (the difference is $7 \%$ ) but as we see later they are larger than the formation times obtained experimentally. The value of $2 T_{0}$ is, therefore, too low.

On the other hand, the choice of $4 T_{0}$ results in the situation where at the onset of stage II the energy of the ring is already larger than the energy provided by the plume and the propagation velocity of the ring exceeds the flow velocity in the stem by more than $30 \%$, thus showing clearly that a duration of $4 T_{0}$ is too large.

Experimental verification of the model can be obtained from the work of Johari \& Gharib (1998) who studied vortex ring formation in a column of negatively buoyant fluid released from a cylindrical tube. Two tube diameters of $0.95 \mathrm{~cm}$ and $1.9 \mathrm{~cm}$, two density differences of $5 \%$ and $9.7 \%$, and several aspect ratios of the buoyant column were considered, heavier fluid being used with the larger tube. Applying the present model and taking the radius of the tube as $R_{0}$ pinch-off is found to take place at about $0.45 \mathrm{~s}$ for both tubes because the characteristic time (1) is the same in both cases. On the other hand, no dependence of the pinch-off time on the aspect ratio is predicted by the theory.

Johari \& Gharib (1998) did not report the exact time of pinch-off, but by using their photographs of buoyant column development and their measurements of the vorticity field we can estimate a time range during which pinch-off occurred. First, on comparing their figures 3 and 4 there is indeed seen to be no dependence on the aspect ratio. Figure 2(a) of Johari \& Gharib (1998) implies that the ring had already pinched off at $0.6 \mathrm{~s}$, while according to their figures $3(a)$ and $4(a)$ pinch-off occurred before $0.57 \mathrm{~s}$ and $0.67 \mathrm{~s}$, respectively. On the other hand, their figure $5(a)$ shows that the ring has not yet pinched off at $0.27 \mathrm{~s}$. Therefore, the plausible time range for pinch-off is $0.3-0.6 \mathrm{~s}$.

We note that the predictions of the model are in agreement with Johari \& Gharib's (1998) results. On the other hand, if one chooses $2 T_{0}$ for the first-stage duration, the prediction for the pinch-off time will be $0.65 \mathrm{~s}$, i.e. too large a value.

Professor H. Johari (personal communication) has informed us that, according to his measurements of the vorticity field at $0.47 \mathrm{~s}$, pinch-off occurred shortly before this time. Hence, the possible time of pinch-off is $0.4-0.45 \mathrm{~s}$, compatible with the predictions of the model.

Using the technique of particle image velocimetry, Pottebaum et al. (1999) studied the pinch-off process of a starting thermal plume. They reported approximate formation number values in the range of 4.7-5. Their detailed experimental results will be reported in a separate paper. 
Finally, it should be noted that Johari \& Gharib (1998) observed that the plume tends to overtake the ring, breaking it up and re-forming into a larger thermal. One of the possible reasons for this phenomenon may be the fact that after pinch-off the buoyant vortex ring starts to expand and slow down (Turner 1957). This phenomenon is beyond the scope of the present study.

\section{Conclusions}

We have showed that the vortex ring formation in a starting buoyant plume takes place in accordance with the Kelvin-Benjamin variational principle: the vortex ring grows as long as the plume is able to provide the energy required for steady vortex ring existence. This generalizes the result obtained by Gharib et al. (1998) for piston-generated vortex rings.

One can reasonably predict the pinch-off time by assuming that at pinch-off the plume and ring velocities are equal.

Available experimental results confirm the predictions of the model.

M. Shusser gratefully acknowledges the financial support provided by Lester Deutsch postdoctoral fellowship. We would like to thank Professor Johari for his critical review of this manuscript and providing some unpublished data.

\section{REFERENCES}

Amick, C. J. \& Fraenkel, L. E. 1986 The uniqueness of Hill's spherical vortex. Arch. Rat. Mech. Anal. 92, 91-119.

BENJAmin, T. B. 1976 The alliance of practical and analytical insights into the nonlinear problems of fluid mechanics. In Application of Methods of Functional Analysis to Problems in Mechanics (ed. P. Germain \& B. Nayroles). Lecture Notes in Mathematics, vol. 503, pp. 8-28. Springer.

Berezin, Y. A. \& Hutter, K. 1995 On vortex air motions above an axisymmetric source of mass, momentum and heat. J. Fluid Mech. 290, 299-317.

Bush, J. W. M. \& Woods, A. W. 1999 Vortex generation by line plumes in a rotating stratified fluid. J. Fluid Mech. 388, 289-313.

Chay, A. \& Shlien, D. J. 1986 Scalar field measurements of a laminar starting plume cap using digital processing of interferograms. Phys. Fluids 29, 2358-2366.

Fage, A. \& Johansen, F. C. 1928 The structure of vortex sheets. Phil. Mag. (7) 5, 417-441.

FAY, J. A. 1973 Buoyant plumes and wakes. Ann. Rev. Fluid Mech. 5, 151-160.

Fernando, H. J. S., Chen, R.-R. \& Ayotte, B. A. 1998 Development of a point plume in the presence of background rotation. Phys. Fluids 10, 2369-2383.

Fraenkel, L. E. 1972 Examples of steady vortex rings of small cross-section in an ideal fluid. J. Fluid Mech. 51, 119-135.

Friedl, M. J., HäRTel, C. \& FAnNeløp, T. K. 1999 An experimental study of starting plumes over area sources. Il Nuovo Cimento C 22, 835-845.

Friedman, A. \& Turkington, B. 1981 Vortex rings existence and asymptotic estimates. Trans. Am. Math. Soc. 268, 1-37.

Gebhart, B., Hilder, D. S. \& Kelleher, M. 1984 The diffusion of turbulent buoyant jets. Adv. Heat Transfer 16, 1-57.

Gharib, M., Rambod, E. \& Shariff, K. 1998 A universal time scale for vortex ring formation. J. Fluid Mech. 360, 121-140.

Johari, H. \& Gharib, M. 1998 Near field development of starting buoyant flows. In Proc. 8th Intl Symp. on Flow Visualization, Sorrento, Italy (ed. G. M. Carlomagno \& I. Grant), pp. 83.1-83.7. Kelvin, Lord 1880 Vortex statics. Phil. Mag. (5) 10, 97-109.

Lim, T. T. \& Nickels, T. B. 1995 Vortex rings. In Fluid Vortices (ed. S. I. Green), pp. 95-153. Kluwer. List, E. J. 1982 Turbulent jets and plumes. Ann. Rev. Fluid Mech. 14, 189-212.

Lundgren, T. S. \& Mansour, N. N. 1991 Vortex ring bubbles. J. Fluid Mech. 224, 177-196. 
Lundgren, T. S., Yao, J. \& Mansour, N. N. 1992 Microburst modelling and scaling. J. Fluid Mech. 239, 461-488.

Mohseni, K. \& Gharib, M. 1998 A model for universal time scale of vortex ring formation. Phys. Fluids 10, 2436-2438.

Morton, B. R., TAYlor, G. \& Turner, J. S. 1956 Turbulent gravitational convection from maintained and instantaneous sources. Proc. R. Soc. Lond. A 234, 1-23.

Moses, E., Zocchi, G. \& Libchaber, A. 1993 An experimental study of laminar plumes. J. Fluid Mech. 251, 581-601.

Norbury, J. 1973 A family of steady vortex rings. J. Fluid Mech. 57, 417-431.

Pottebaum, T., Shusser, M. \& Gharib, M. 1999 Vortex ring formation in a starting buoyant plume. Bull. Am. Phys. Soc. 44, 140.

Rast, M. P. 1998 Compressible plume dynamics and stability. J. Fluid Mech. 369, 125-149.

SARPKaYA, T. 1975 Vortex shedding from inclined plate. J. Fluid Mech. 68, 109-128.

Shariff, K. \& Leonard, A. 1992 Vortex rings. Ann. Rev. Fluid Mech. 24, 253-279.

Shlien, D. J. \& Boxman, R. L. 1981 Laminar starting plume temperature field measurement. Intl J. Heat Mass Transfer 24, 919-931.

Shusser, M. \& GHarib, M. 2000 Energy and velocity of a forming vortex ring. Phys. Fluids 12, 618-621.

Tanny, J. \& Shlien, D. J. 1985 Velocity field measurements of a laminar starting plume. Phys. Fluids 28, 1027-1032.

Turner, J. S. 1957 Buoyant vortex rings. Proc. R. Soc. Lond. A 239, 61-75.

Turner, J. S. 1962 The 'starting plume' in neutral surroundings. J. Fluid Mech. 13, 356-368.

TURner, J. S. 1973 Buoyancy Effects in Fluids. Cambridge University Press.

WAN, Y.-H. 1988 Variational principles for Hill's spherical vortex and nearly spherical vortices. Trans. Am. Math. Soc. 308, 299-312. 